\title{
GPRC5A exerts its tumor-suppressive effects in breast cancer cells by inhibiting EGFR and its downstream pathway
}

\author{
LU YANG ${ }^{1-3 *}$, TAO MA ${ }^{1-3^{*}}$ and JIN ZHANG ${ }^{1-3}$ \\ ${ }^{1}$ The Third Surgical Department of Breast Cancer, Tianjin Medical University Cancer Institute and Hospital; \\ ${ }^{2}$ Tianjin Medical University Cancer Institute and Hospital, National Clinical Research Center of Cancer; \\ ${ }^{3}$ Key Laboratory of Breast Cancer Prevention and Therapy, Tianjin Medical University, \\ Chinese Ministry of Education, He Xi, Tianjin 300060, P.R. China
}

Received November 30, 2015; Accepted January 5, 2016

DOI: $10.3892 /$ or.2016.5062

\begin{abstract}
G-protein-coupled receptor family $\mathrm{C}$ group 5 member A (GPRC5A) is a member of the type 3-G protein-coupling receptor family. Previous studies have observed dysregulated expression of GPRC5A in several malignant diseases which suggests that GPRC5A may participate in tumor progression. However, these results are controversial. In the present study, we explored the biological functions of GPRC5A in breast cancer cells and aimed to uncover the related molecular mechanisms. Our results demonstrated that GPRC5A is a tumor suppressor in EGFR-expressing breast cancer cells. GPRC5A knockdown in EGFR-expressing MDA-MB-231 cells promoted colony formation, cell proliferation, cell migration and invasion, while GPRC5A suppression had no impact on MCF7 cells, which express no EGFR. Furthermore, GPRC5A knockdown enhanced EGF-induced EGFR and its downstream signaling activation. Inhibition of EGFR phosphorylation in MDA-MB-231 cells suppressed the tumorigenic effects of GPRC5A knockdown. Moreover, GPRC5A knockdown exhibited an oncogenic role in MCF7 cells transfected with the EGFR-expressing plasmid. Taken together, our results implicate GPRC5A as a tumor suppressor in breast cancer cells, and GPRC5A exerts its tumor-suppressive function by inhibiting EGFR and its downstream pathway.
\end{abstract}

\section{Introduction}

Breast cancer is one of the most common malignancies worldwide. In the US, it is estimated that 234,190 patients were

Correspondence to: Dr Jin Zhang, The Third Surgical Department of Breast Cancer, Tianjin Medical University Cancer Institute and Hospital, Huan-Hu-Xi Road, Ti-Yuan-Bei, He Xi, Tianjin 300060, P.R. China

E-mail: zhangjintmu@gmail.com; mt19861029@126.com

*Contributed equally

Key words: GPRC5A, epidermal growth factor receptor, triplenegative breast cancer, breast cancer diagnosed with breast cancer and 40,730 succumbed to the disease in 2015 (1). Breast cancer is a heterogeneous disease. In addition, this heterogeneity occurs both between tumors (inter-tumor heterogeneity) and within tumors (intra-tumor heterogeneity). This high diversity determines the danger of disease progression and challenges the effectiveness of treatment strategies $(2,3)$. Therefore, in-depth understanding of the heterogeneity of the disease may elucidate strategies by which to conquer the disease.

Triple-negative breast cancer (TNBC) is a subtype of invasive breast cancer with an estrogen receptor-negative (ER-), progesterone receptor-negative ( $\left(\mathrm{PR}^{-}\right)$, and HER-2-negative (HER2) phenotype. It is a significant and noticeable subtype of breast cancer due to its relationship with basal-like breast cancer (4). Although TNBC is highly proliferative and sensitive to systemic chemotherapies, patient outcomes are poor compared with other subtypes of breast cancer $\left(\mathrm{HER} 2^{+}\right.$or $\left.\mathrm{ER}^{+}\right)(5-7)$. TNBC is the only major type of breast cancer for which no specific FDA-approved targeted therapy is available to improve patient outcomes; it is resistant to targeted therapies such as hormonal and HER2-targeting therapies (6).

The epidermal growth factor receptor (EGFR), a cellsurface receptor, which is a member of the ErbB family of tyrosine kinases, plays a critical role in the regulation of cell proliferation, survival and differentiation (8). Dysregulation of EGFR has been reported in a wide variety of carcinomas, including head and neck, breast, bladder, ovarian, renal, colon and lung cancer (8-10). In breast cancer, abnormal activation of EGFR is associated with aggressive phenotypes, such as large tumor size, poor differentiation and poor clinical outcomes (11-13). Moreover, overexpression and activation of EGFR have been frequently reported in TNBC and provide a potential target for TNBC $(14,15)$. Therefore, a full understanding of the EGFR pathway in TNBC is a precondition for conquering the disease.

G-protein-coupled receptor family $\mathrm{C}$ group 5 member $\mathrm{A}$ (GPRC5A) is a member of the type 3-G protein-coupling receptor family, characterized by the signature 7-transmembrane domain motif. Its role in cancer was first unveiled in lung cancer and it has been recognized as a growth-promoting gene and a novel P53 transcriptional target (16). Further study showed that GPRC5A was downregulated in $61 \%$ of lung 
tumors compared with adjacent normal tissues (17). Results from Gprc5a-knockout mice suggest a tumor-suppressive role in lung adenocarcinoma (18). Molecular and pathway analysis indicates interaction between EGFR and GPRC5A and negative regulation of EGFR by GPRC5A $(19,20)$. In breast cancer, a high prevalence of GPRC5A germline mutations was found in BRCA1-mutant breast cancer patients (21). However, the exact biological functions of GPRC5A and its correlation with EGFR in breast cancer remain obscure. In the present study, we explored the functional role of GPRC5A and the related molecular mechanisms in breast cancer cells in an attempt to discover useful information and clues for developing a novel treatment strategy for TNBC.

\section{Materials and methods}

Chemicals, cell culture and plasmid transfection. Breast cancer cell lines MDA-MB-231 and MCF7 were obtained from the Type Culture Collection of the Chinese Academy of Sciences and were maintained in Dulbecco's modified Eagle's medium (DMEM) with 10\% fetal bovine serum (FBS) in a $5 \% \mathrm{CO}_{2}$-humidified, $95 \%$ air incubator at $37^{\circ} \mathrm{C}$. A plasmid expressing EGFR (plasmid \#11011) was obtained from Addgene (Cambridge, MA, USA). Transient transfection was performed using the Lipofectamine 2000 reagent (Invitrogen, Carlsbad, CA, USA) following the manufacturer's instructions. MEK inhibitor, PD98059, was obtained from Cell Signaling Technology (Danvers, MA, USA).

Lentiviral infection. GPRC5A-shRNA and the control lentivirus were obtained from GenePharma (Shanghai, China). The GPRC5A-shRNA1 target sequence was: 5'-GCTTATGTTA GTCCCGAGTTT-3'; and the GPRC5A-shRNA2 target sequence was: 5'-CCTGACCATGAATAGGACCAA-3'. The virus-containing supernatant was incubated on target cells for $12 \mathrm{~h}$ with $8 \mu \mathrm{g} / \mathrm{ml}$ Polybrene, following the manufacturer's instructions. Infected cells were selected in puromycin, as optimized for each cell line.

Colony formation assay. MDA-MB-231 and MCF7 cells infected with GPRC5A-shRNA1, GPRC5A-shRNA2 or the scramble were plated into a 6 -well plate $(1,000$ cells/well). Medium was replaced every 2 days, and the cells were cultured for 2 weeks in a $5 \% \mathrm{CO}_{2}$ environment at $37^{\circ} \mathrm{C}$. Then, the colonies were stained with $0.01 \%$ (w/v) crystal violet. Colonies containing $>50$ cells were counted.

Apoptosis and BrdU incorporation assay. Apoptosis rates were measured using the PE Annexin $\mathrm{V}$ apoptosis detection kit (BD Pharmingen, San Diego, CA, USA) following the manufacturer's instructions. Briefly, MCF7 and MDA-MB-231 cells were incubated with cisplatin $(40 \mu \mathrm{M})$ for $24 \mathrm{~h}$, and then trypsinized and resuspended in $100 \mu \mathrm{l}$ binding buffer. Annexin V and PI ( $5 \mu \mathrm{l})$ were added to each tube. Then, $400 \mu \mathrm{l}$ binding buffer was added to each reaction tube and the cells were analyzed by flow cytometry. Cell proliferation rates were determined with the BrdU incorporation assay using cell proliferation ELISA kit (Roche). The measurements were performed following the manufacturer's instructions as previously described (22).
$R N A$ extraction and $q R T-P C R$. Total RNA was isolated using TRIzol reagent (Invitrogen) following the manufacturer's instructions. Total RNA $(2 \mu \mathrm{g})$ was used for the synthesis of first-strand cDNA using a High Capacity cDNA reverse transcription kit (Invitrogen, Beijing, China). Quantitative real-time PCR was performed using SYBR-Green Mix (Applied Biosystems, Foster City, CA, USA). The reactions were performed using a 7500 Fast Real-Time PCR system (Applied Biosystems). The data are displayed as $2^{-\Delta \Delta C t}$ values and are representative of at least 3 independent experiments. Primers used in the present study were: GPRC5a, ATGGCTA CAACAGTCCCTGAT and CCACCGTTTCTAGGACGA TGC; EGFR, AGGCACGAGTAACAAGCTCAC and ATGA GGACATAACCAGCCACC.

CCK-8 assay. MCF7 $(8,000)$ and MBA-MD-231 $(5,000)$ cells were placed into 96 -well plates. The cells were continually cultured for 24-72 h. At 24, 48 and $72 \mathrm{~h}, 10 \mu \mathrm{l}$ of Cell Counting Kit-8 (CCK-8) reagent (Dojindo, Shanghai, China) was added to each well. Then, the cells were incubated at $37^{\circ} \mathrm{C}$ for another $2 \mathrm{~h}$. After shaking for $20 \mathrm{~min}$, the absorbance was detected at $450 \mathrm{~nm}$ on a microplate spectrophotometer (Bio-Tek Instruments, Winooski, VT, USA).

Migration and invasion assays. For the Transwell migration assay, the breast cancer cells were trypsinized and placed in the upper chamber of each insert (Corning, Cambridge, MA, USA, USA) containing the non-coated membrane. For the invasion assay, the cells were placed on the upper chamber of each insert coated with $40 \mu \mathrm{l}$ of Matrigel (BD Biosciences, Franklin Lakes, NJ, USA), which was diluted to $4 \mu \mathrm{g} / \mu 1$ with serum-free medium. Then, the medium supplemented with $20 \%$ FBS $(600 \mu \mathrm{l})$ was added to the lower chambers. After $24 \mathrm{~h}$ of incubation at $37^{\circ} \mathrm{C}$, the upper surface of the membrane was wiped with a cotton tip, and the cells that had attached to the lower surface were stained for $10 \mathrm{~min}$ with crystal violet. Cells in 5 random fields of view at a magnification of $x 100$ were counted and values are expressed as the average number of cells/field of view. All of the assays were performed in triplicate.

Western blot assay. Whole-cell protein extracts were obtained using RIPA buffer (Beyotime Institute of Biotechnology, Nanjing, China). Then, the extracts were separated on $10 \%$ SDS denatured polyacrylamide gel electrophoresis (PAGE) gels, transferred to nitrocellulose membranes and blocked in phosphate-buffered saline/Tween-20 containing 5\% non-fat milk. The membranes were incubated with the antibodies overnight at $4^{\circ} \mathrm{C}$. The membranes were then incubated with the HRP-labeled corresponding IgG for $1 \mathrm{~h}$. The protein expression level was assessed by enhanced chemiluminescence and exposure to film (Fujifilm, Tokyo, Japan). The anti-GPRC5A antibody (cat. no. HPA007928) was obtained from Sigma-Aldrich (Shanghai, China). The anti-E-cadherin (ab15148), anti- $\beta$-actin (ab8229), anti-EGFR (ab2430) and anti-phospho-EGFR (ab40815) antibodies were obtained from Abcam (Cambridge, MA, USA). The anti-Akt (9272), anti-phospho-Akt (9271), anti-Stat3 (9132) and anti-phosphoStat3 (9131) antibodies were purchased from Cell Signaling Technology. 
A

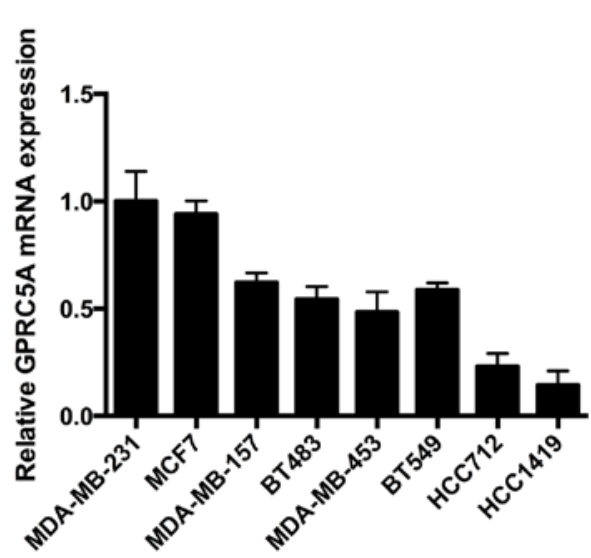

C

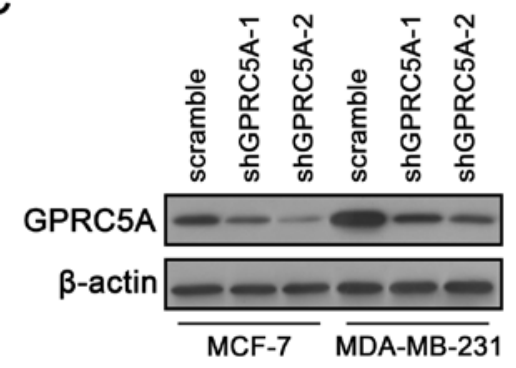

D

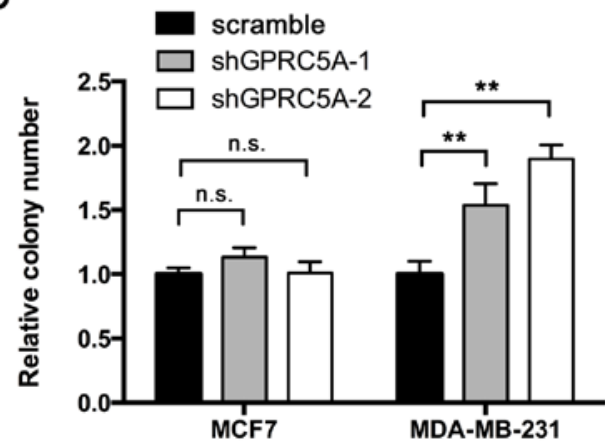

B

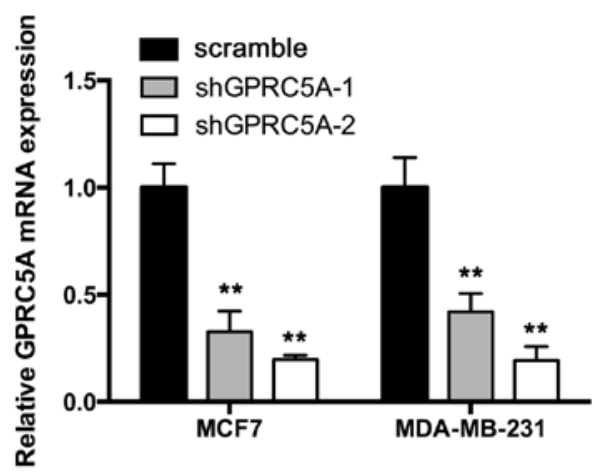

E

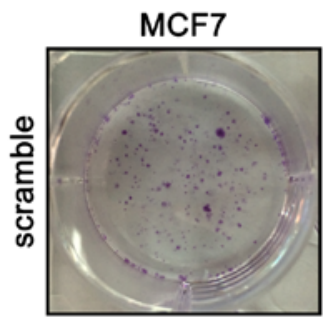

MDA-MB-231
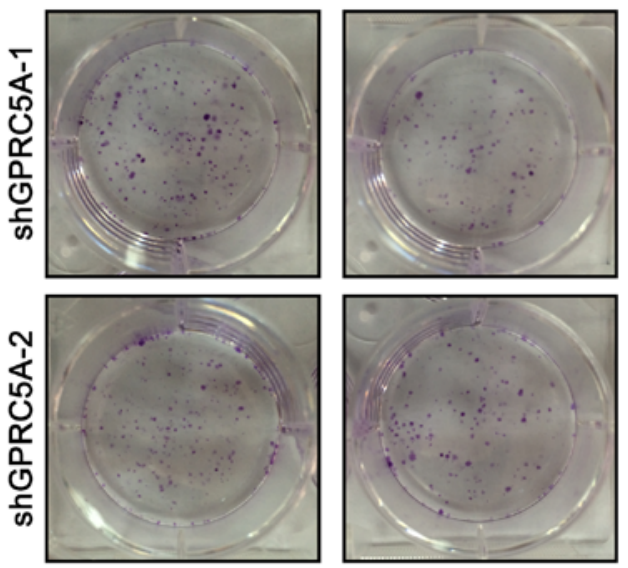

Figure 1. GPRC5A knockdown promotes colony formation in MDA-MB-231 cells but not in MCF7 cells. (A) GPRC5A expression was determined by RT-qPCR assay in 8 breast cell lines. (B and C) MCF7 and MDA-MB-231 cells were infected with a lentivirus targeting GPRC5A (shGRPC5A-1 and -2), or scramble. Then, GRPC5A knockdown efficiency was determined by RT-qPCR and western blot assay. (D and E) MCF7 and MDA-MB-231 cells infected with shGPRC5A-1 and -2, or scramble were subjected to colony formation assay. n.s., not significant; ${ }^{* *} \mathrm{P}<0.01$. Columns, mean; bars, SD.

Statistical analysis. Experimental results are expressed as mean \pm standard deviation (SD). Statistically significant differences between groups were determined using a two-tailed unpaired Student's t-test. All statistical analyses were performed using SPSS 16.0 software (SPSS, Inc., Chicago, IL, USA). $\mathrm{P}<0.05$ was considered to indicate a statistically significant result.

\section{Results}

GPRC5A knockdown promotes colony formation in $M D A-M B-231$ cells but not in MCF7 cells. To investigate its role in breast cancer, we first examined GPRC5A expression in established breast cancer cell lines (Fig. 1A). We chose high GPRC5A-expressing cell lines, MCF7 and MDA-MB231, for further investigation. Expression of GPRC5A in both cell lines was suppressed using short hairpin RNAs
(shRNAs), which targeted 2 different sequences in RASAL2. RT-qPCR and western blot assay were performed to confirm the knockdown efficiency. As shown in Fig. 1B, the expression of GPRC5A mRNA and protein were inhibited in cells transfected with shRNA-GPRC5A-1 or shRNA-GPRC5A-2 compared with scramble shRNA. Then a colony formation assay was performed. The results showed that the colony number was unaffected by GPRC5A knockdown in the MCF7 cells (Fig. 1D and E). However, MDA-MB-231 cells transfected with GPRC5A shRNA produced more colonies than the control cells in the colony formation assay (Fig. 1D and E). These results indicate that GPRC5A may play a suppressive role in breast cancer cells.

GPRC5A knockdown enhances cell proliferation in MDA-MB-231 cells butnot in MCF 7 cells. To further investigate the significance of GPRC5A in breast cancer, we determined 
A

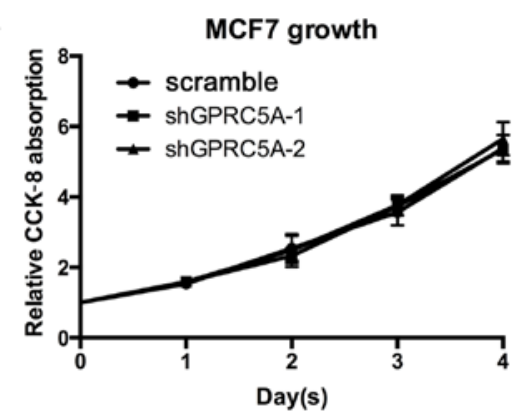

C

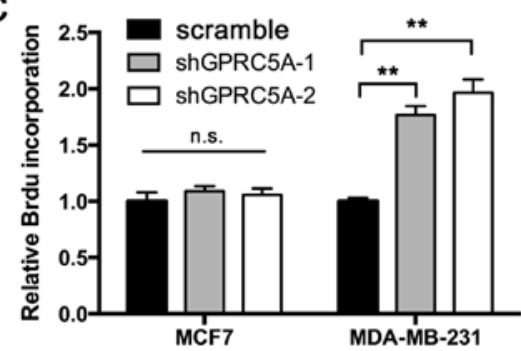

D

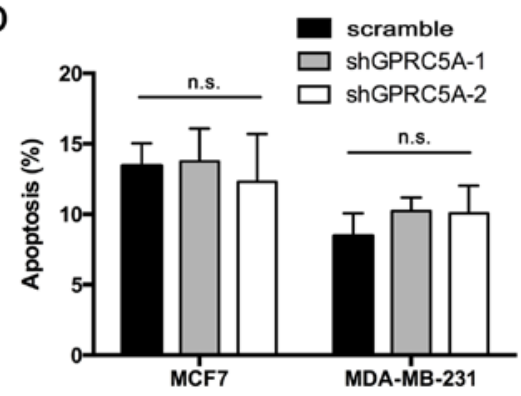

B

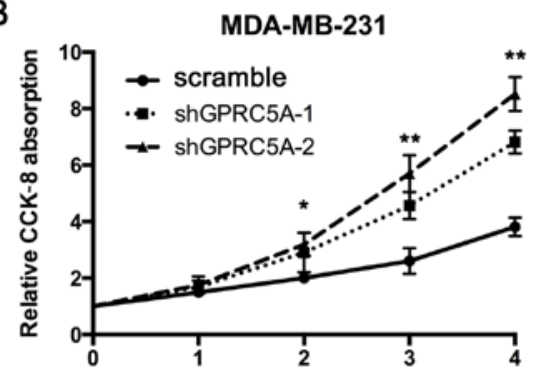

$E$
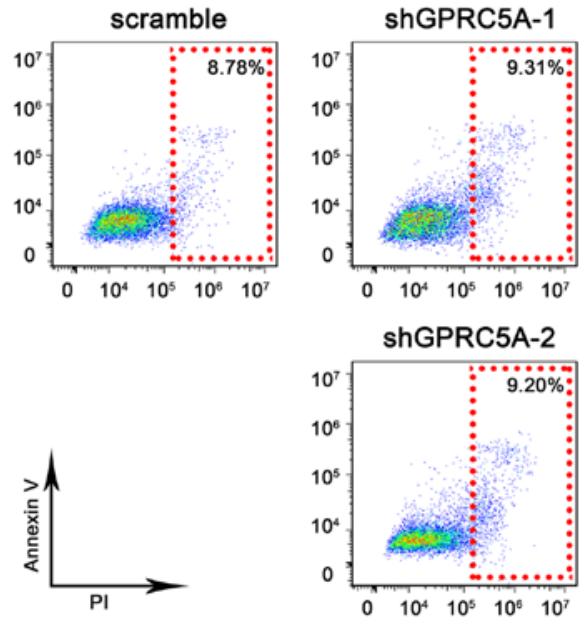

Figure 2. GPRC5A knockdown enhances cell proliferation in MDA-MB-231 cells but not in MCF7 cells. (A and B) To examine cell proliferation, MCF7 and MDA-MB-231 cells infected with shGPRC5A-1 and -2, or scramble were subjected to CCK-8 assay. (C) BrdU incorporation assay was performed to confirm the previous results. (D and E) Apoptosis rates were determined using Annexin V and PI assay. n.s., not significant; ${ }^{*} \mathrm{P}<0.05$, ${ }^{* *} \mathrm{P}<0.01$. Columns, mean; bars, SD.

A

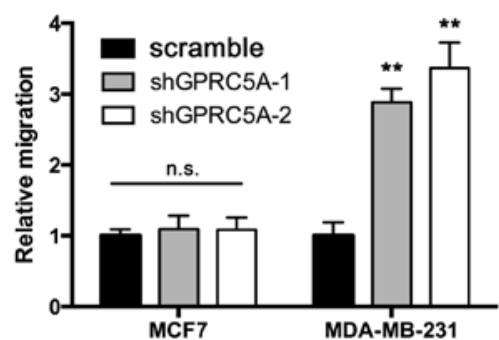

B

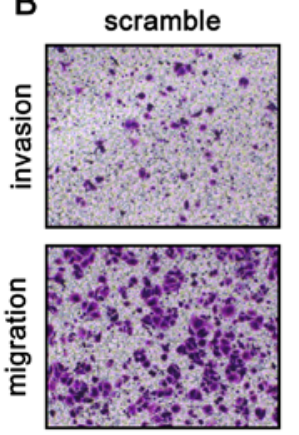

shGPRC5A-1
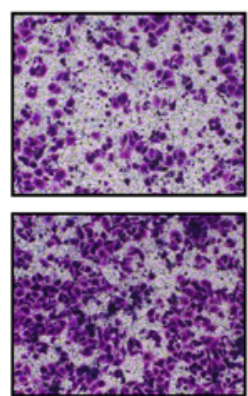

ShGPRC5A-2

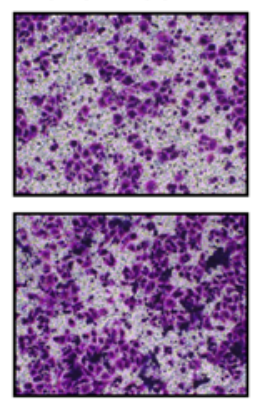

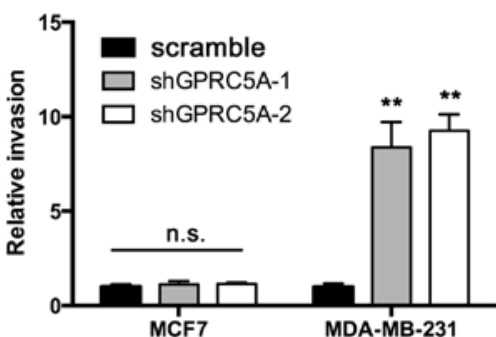

C

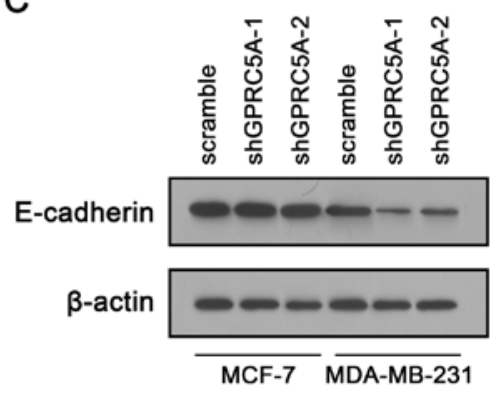

Figure 3. GPRC5A knockdown promotes migration and invasion in the MDA-MB-231 cells. (A and B) MCF7 and MDA-MB-231 cells infected with shGPRC5A-1 and -2, or scramble were subjected to in vitro migration and invasion assays. Cells in 5 random fields of view at a magnification of x100 were counted and expressed as the average number of cells/field of view. (C) E-cadherin expression in MCF7 and MDA-MB-231 cells infected with shGPRC5A-1 and -2 , or scramble was determined by western blot assay. n.s., not significant; ${ }^{* *} \mathrm{P}<0.01$. Columns, mean; bars, SD. 
whether GPRC5A affects breast cancer cell proliferation and apoptosis. We first performed the CCK- 8 assay to construct a proliferation curve. As shown in Fig. 2A, although GPRC5A knockdown had no impact on MCF7 proliferation, MDA-MB231 cells exhibited accelerated proliferation after GPRC5A knockdown (Fig. 2B). To further validate the role of GPRC5A in proliferation, we performed BrdU incorporation assay. Consistent with the previous results, GPRC5A knockdown aggravated BrdU incorporation in the MDA-MB-231 cells, while BrdU uptake remained unaffected in the MCF7 cells (Fig. 2C). Furthermore, we examined whether GPRC5A knockdown controls cell apoptosis. The results showed that GPRC5A knockdown did not regulate apoptosis in the MCF7 and MDA-MB-231 cells (Fig. 2D and E). Taken together, these results confirmed the suppressive role of GPRC5A in MDA-MB-231 cells, particularly in regards to proliferation.

GPRC5A knockdown promotes migration and invasion in $M D A-M B-231$ cells. Migration and invasion are critical steps in the initial process of cancer metastasis (23). To further investigate the significance of GPRC5A downregulation in breast cancer, we determined whether inhibition of GPRC5A also promotes migration and invasion. A Transwell migration assay was performed to assess migratory ability. The results showed that inhibition of GPRC5A significantly increased MDA-MB-231 cell migration. However, the migratory ability of the MCF7 cells was unaltered after GPRC5A knockdown (Fig. 3A and B). We further confirmed these findings using a wound healing assay. Consistent with the data obtained from the Transwell migration assay, GPRC5A downregulation significantly promoted the migration of the MDA-MB-231 cells, but had no impact on MCF7 cell migration. In addition, we used a Matrigel-coated Transwell to assess cell invasive ability. The MDA-MB-231 cells exhibited increased invasive ability after GPRC5A knockdown. In agreement with the previous results, MCF7 cells transfected with GPRC5A shRNA showed no increased invasive ability when compared with that noted in the control cells (Fig. 3A and B). E-cadherin is a key player in the regulation of cancer cell EMT and metastasis (24). We further determined whether GPRC5A participates in the regulation of E-cadherin. RT-qPCR and western blot assay were performed. The results showed that GPRC5A downregulation suppressed GPRC5A expression in the MDA-MB-231 cells but not in the MCF7 cells (Fig. 3C). Taken together, our results showed that GPRC5A may be a potential metastatic inhibitor in breast cancer.

GPRC5A knockdown enhances EGFR activation in the MDA-MB-231 cells but not in the MCF7 cells. The previous results confirmed the tumor-suppressive role of GPRC5A in MDA-MB-231 cells. Notably, GPRC5A did not exhibit any tumor-suppressive effects in the MCF7 cells. MDA-MB-231 cells express EGFR, while MCF7 do not. We aimed to ascertain whether GPRC5A exerts its tumor-suppressive effects through EGFR. First, we confirmed its expression in the MCF7 and MDA-MB-231 cells. We did not detect EGFR expression in the MCF7 cells, while the MDA-MB-231 cells exhibited relatively strong expression (Fig. 4A). Then, we treated MDA-MB-231 cells infected with GPRC5A shRNA or scramble shRNA with EGF for $4 \mathrm{~h}$ and examined EGFR activity. The results
A

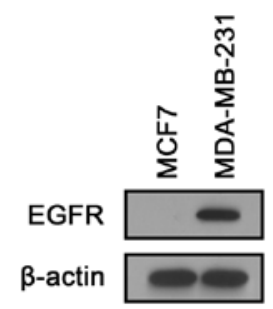

B

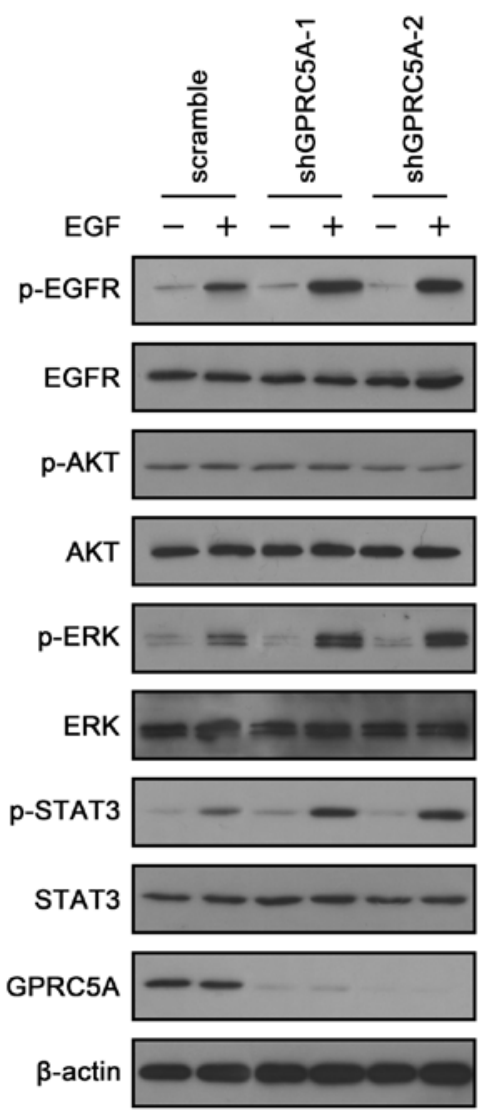

C

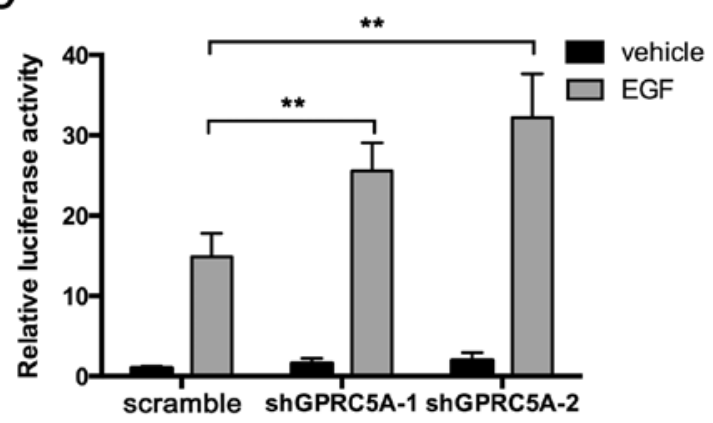

Figure 4. GPRC5A knockdown enhances EGFR activation in MDA-MB-231 cells but not in MCF7 cells. (A) EGFR expression in the MCF7 and MDA-MB-231 cells was determined by western blot assay. (B) MCF7 and MDA-MB-231 cells infected with shGPRC5A-1 and -2 , or scramble were incubated with EGF or vehicle for $2 \mathrm{~h}$. Then, expression of the indicated proteins was determined by western blot assay. (C) STAT3 luciferase reporter assay was performed to examine EGF-induced STAT3 activation. n.s., not significant; ${ }^{* *} \mathrm{P}<0.01$. Columns, mean; bars, $\mathrm{SD}$.

showed that GPRC5A knockdown significantly enhanced EGF-induced EGFR activation (Fig. 4B). In addition, 
A

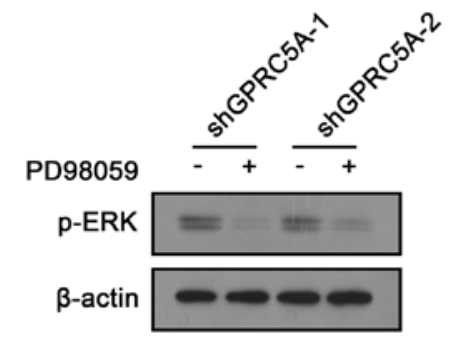

B

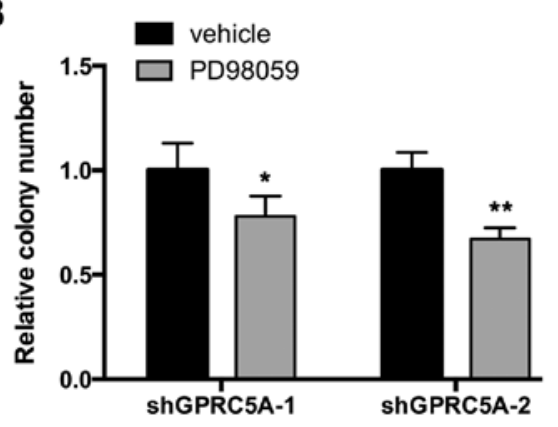

D

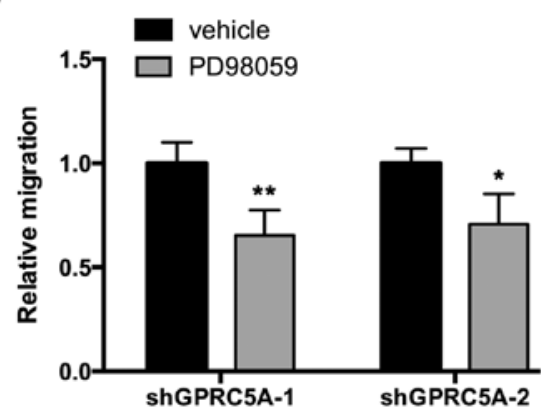

C

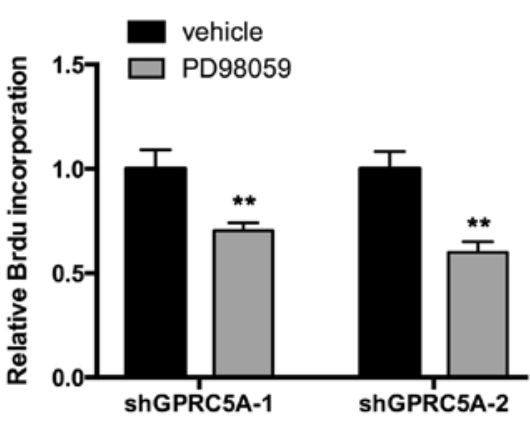

E

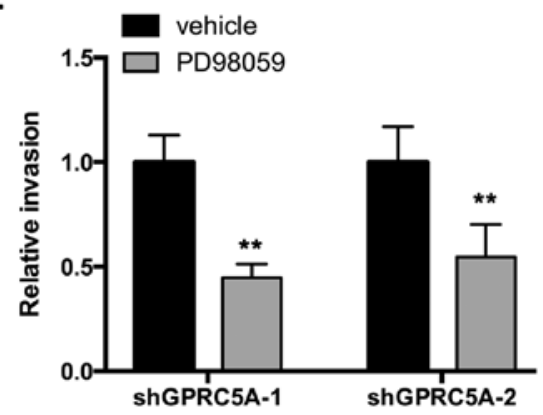

Figure 5. PD98059 inhibits the oncogenic effects of GPRC5A knockdown in MDA-MB-231 cells. (A) MDA-MB-231 cells infected with shGPRC5A-1 or -2 were incubated with PD98059 or vehicle for $12 \mathrm{~h}$, and then expression of phospho-ERK was examined by western blot assay. (B) Colony formation, (C) BrdU incorporation and ( $\mathrm{D}$ and $\mathrm{E}$ ) in vitro migration and invasion assays were also performed. n.s., not significant; ${ }^{*} \mathrm{P}<0.05,{ }^{* *} \mathrm{P}<0.01$. Columns, mean; bars, $\mathrm{SD}$.

p-STAT3 was also increased in the GPRC5A-knockdown cells compared with that noted in the control cells (Fig. 4B). ERK is one of the key downstream effectors of EGFR. Consistent with EGFR and STAT3, total expression of ERK was not altered, while p-ERK was significantly increased after EGF treatment in the GPRC5A-knockdown cells (Fig. 4B). To further validate the role of GPRC5A in EGF-induced EGFR activation, we performed STAT3 luciferase reporter assay. MDA-MB-231 cells infected with GPRC5A shRNA or scramble shRNA were transfected with STAT3 luciferase and treated with EGF for 4 h. GPRC5A inhibition enhanced STAT3 luciferase activity in the MDA-MB-231 cells (Fig. 4C). Taken together, these results indicate that GPRC5A may participate in the negative regulation of EGFR in breast cancer cells.

PD98059 inhibits the oncogenic effects of GPRC5A knockdown in the MDA-MB-231 cells. ERK is the key downstream effector of EGFR. We aimed to ascertain whether GPRC5A exerts its tumor-suppressive effects through the EGFR-ERK pathway. Thus, we inhibited ERK activation by treating the MDA-MB-231 cells with PD98059, an MEK inhibitor. The inhibition was confirmed by western blot assay (Fig. 5A).
Then, colony formation was performed. As expected, PD98059 suppressed the colony formation of the GPRC5A-knockdown cells (Fig. 5B). Furthermore, BrdU incorporation rate was also decreased after PD98059 treatment (Fig. 5C). Next, we examined whether PD98059 had an impact on the regulation of migration and invasion by GPRC5A. Transwell migration assay showed that PD98059 reversed GPRC5A knockdowninduced enhancement of migration (Fig. 5D). In addition, PD98059 also inhibited the enhanced invasive ability of the GPRC5A-knockdown cells (Fig. 5E). Taken together, these results indicate that GPRC5A exerts its tumor-suppressive effects by inhibiting, at least in part, the EGFR-ERK pathway.

GPRC5A knockdown exhibits oncogenic effects in the MCF7 cells transfected with EGFR. Previous results showed that GPRC5A inhibits EGFR activation, and therefore suppresses breast cancer cell proliferation, migration and invasion. To further confirm this conclusion, we overexpressed EGFR in the MCF7 cells which do not express EGFR. The overexpression was confirmed by RT-qPCR and western blot assay (Fig. 6A and B). Then, the colony formation assay was performed. As shown in Fig. 6C, EGFR overexpression 

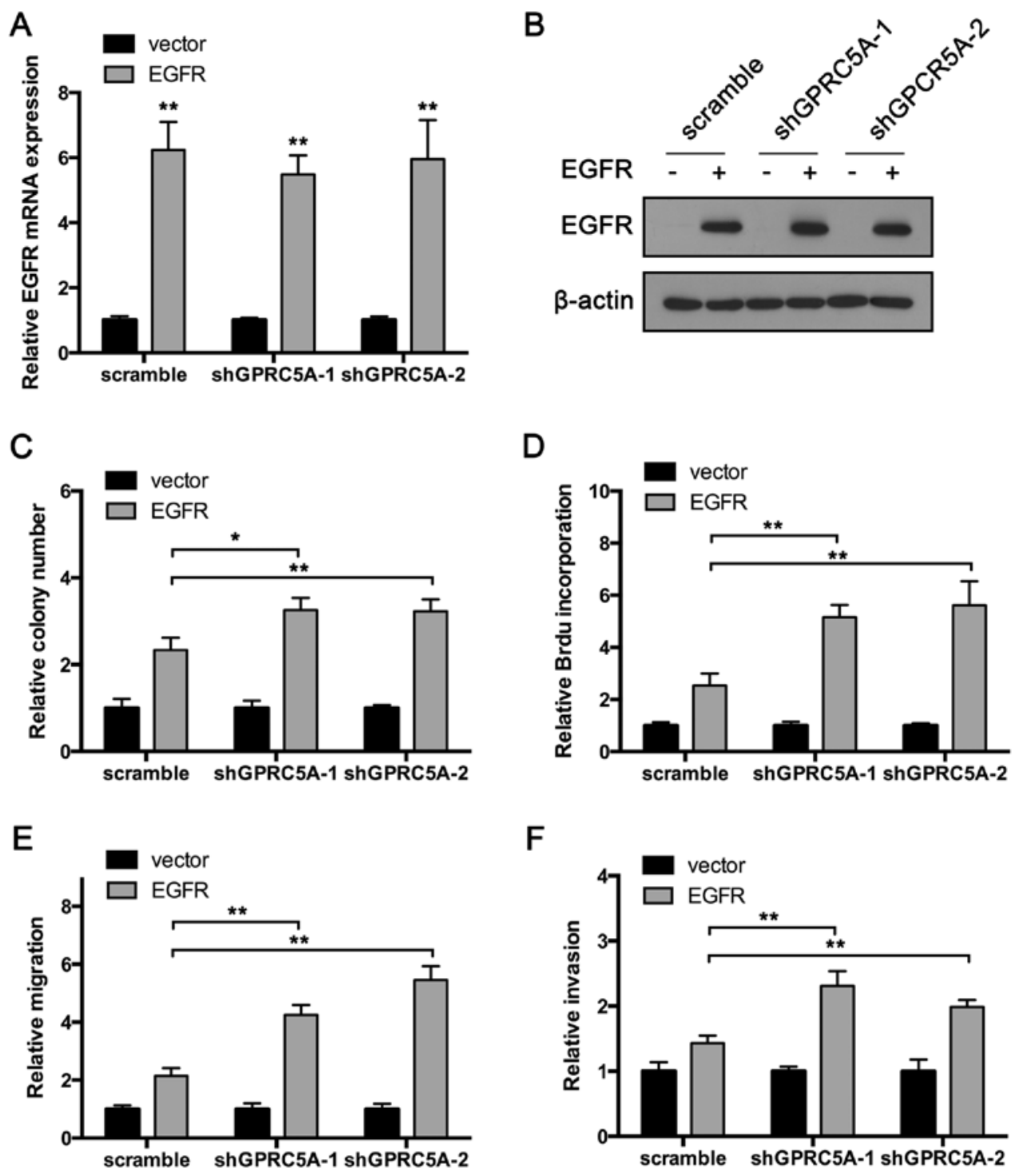

Figure 6. GPRC5A knockdown exhibits oncogenic effects on MCF7 cells transfected with EGFR. MCF7 cells infected with shGPRC5A-1 and -2, or scramble were transfected with the EGFR expression plasmid or control vector. (A and B) EGFR expression efficiency was examined by RT-qPCR and western blot assay. (C) Colony formation, (D) BrdU incorporation and (E and F) in vitro migration and invasion assays were also performed. n.s., not significant; "P<0.05, ${ }^{* * *} \mathrm{P}<0.01$. Columns, mean; bars, SD.

increased the colonies produced by the MCF7 cells. Consistent with the previous experiment, GPRC5A suppression in MCF7 cells transfected with the vector plasmid produced the same number of colonies compared with that noted in the control cells (Fig. 6C). Notably, GPRC5A knockdown significantly increased the colonies produced by EGFR-overexpressing MCF7 cells (Fig. 6C). Furthermore, EGFR overexpression enhanced BrdU incorporation in the MCF7 cells. Consistent with the colony formation assay, GPRC5A inhibition aggravated BrdU incorporation in the MCF7 cells transfected with the EGFR-expressing plasmid, while no impact on the MCF7 cells transfected with the vector plasmid was observed (Fig. 6D).

In addition, we performed Transwell migration and invasion assays. The results showed that EGFR overexpression significantly promoted MCF7 cell migration and invasion. GPRC5A inhibition suppressed the invasion and migration of the MCF7 cells transfected with the EGFR-expressing plasmid (Fig. 6E and F). Taken together, these results indi- cate that GPRC5A exerts its tumor-suppressive functions by targeting EGFR.

\section{Discussion}

GPRC5A was initially discovered as a retinoic acid-inducible gene strongly expressed in the lung $(16,25,26)$. Subsequent study provided evidence for a role of GPRC5A in lung carcinogenesis. The level of GPRC5A mRNA is often lower in lung tumors compared with normal tissues, and a somatic loss of GPRC5A is associated with poor survival of lung cancer patients. GPRC5A-knockout mice are prone to sporadic and carcinogen- or inflammation-induced lung adenocarcinomas strongly suggesting its role as a lung cancer tumor-suppressor gene. In contrast to lung cancer, GPRC5A is frequently overexpressed in myelodysplastic syndrome as well as in breast, colon and gastric cancers. This indicates that GPRC5A may play a pro-survival role at least in some circumstances. 
In the present study, we knocked down GPRC5A expression in the TNBC cell line, MDA-MB-231. The cells produced more and larger colonies when compared with the control cells. Consistent with this result, cell proliferation was also enhanced after GPRC5A suppression. Furthermore, we observed enhanced in vitro migration and invasion in the GPRC5A-downregulated cells. These results implicate GPRC5A as a tumor-suppressor in MDA-MB-231 cells.

Signaling pathway analysis showed that GPRC5A suppression significantly enhanced EGF-induced EGFR pathway activation in the MDA-MB-231 cells. Inhibition of ERK activation reversed the GPRC5A-induced oncogenic effects. However, the oncogenic effects of GPRC5A knockdown were not reproducible in the MCF7 cells. Notably, when the MCF7 cells were transfected with the EGFR-expressing plasmid, GPRC5A suppression enhanced EGFR activation and EGFR induced oncogenic effects. These results indicate that GPRC5A is a downstream regulator of the EGFR pathway in breast cancer.

TNBC refers to any breast cancer that does not express the genes for estrogen receptor (ER), progesterone receptor (PR) or Her2/neu. Thus, TNBC is more difficult to treat since most chemotherapies target one of the three receptors. Consequently, triple-negative cancers often require combinatorial therapies (27). Exploring novel approaches to treat this subtype is critical since $<30 \%$ of women with metastatic breast cancer survive 5 years and virtually all women with metastatic TNBC ultimately die of their disease despite systemic therapy (27). EGRF is frequently overexpressed and has been proposed as a therapeutic target for TNBC $(2,5,8,27,28)$. Our results provide a new regulatory mechanism of the EGFR pathway in TNBC cells.

In summary, we found that GPRC5A functions as a tumor suppressor in breast cancer cells. GPRC5A inhibits cell proliferation, migration and invasion in vitro and exerts its tumor-suppressive functions by inhibiting EGFR and its downstream pathway. Furthermore, our results highlight the potential of targeting EGFR and the ERK pathway and provide a new therapeutic target for TNBC treatment.

\section{References}

1. Siegel RL, Miller KD and Jemal A: Cancer statistics, 2015. CA Cancer J Clin 65: 5-29, 2015.

2. Polyak K: Heterogeneity in breast cancer. J Clin Invest 121: 3786-3788, 2011.

3. Qin XJ and Ling BX: Proteomic studies in breast cancer (Review). Oncol Lett 3: 735-743, 2012.

4. Sun B, Zhang S, Zhang D, Li Y, Zhao X, Luo Y and Guo Y; Identification of metastasis-related proteins and their clinical relevance to triple-negative human breast cancer. Clin Cancer Res 14: 7050-7059, 2008.

5. Teng YH, Tan WJ, Thike AA, Cheok PY, Tse GM, Wong NS, Yip GW, Bay BH and Tan PH: Mutations in the epidermal growth factor receptor (EGFR) gene in triple negative breast cancer: Possible implications for targeted therapy. Breast Cancer Res 13: R35, 2011

6. Ueno NT and Zhang D: Targeting EGFR in triple negative breast cancer. J Cancer 2: 324-328, 2011.

7. Liu M, Mo QG, Wei CY, Qin QH, Huang Z and He J: Platinumbased chemotherapy in triple-negative breast cancer: A meta-analysis. Oncol Lett 5: 983-991, 2013.
8. Yewale C, Baradia D, Vhora I, Patil S and Misra A: Epidermal growth factor receptor targeting in cancer: A review of trends and strategies. Biomaterials 34: 8690-8707, 2013.

9. Herbst RS: Review of epidermal growth factor receptor biology. Int J Radiat Oncol Biol Phys 59 (Suppl 2): 21-26, 2004.

10. Tomas A, Futter CE and Eden ER: EGF receptor trafficking: Consequences for signaling and cancer. Trends Cell Biol 24: 26-34, 2014.

11. Masuda H, Zhang D, Bartholomeusz C, Doihara $\mathrm{H}$, Hortobagyi GN and Ueno NT: Role of epidermal growth factor receptor in breast cancer. Breast Cancer Res Treat 136: 331-345, 2012.

12. Bhargava R, Gerald WL, Li AR, Pan Q, Lal P, Ladanyi M and Chen B: EGFR gene amplification in breast cancer: Correlation with epidermal growth factor receptor mRNA and protein expression and HER-2 status and absence of EGFR-activating mutations. Mod Pathol 18: 1027-1033, 2005.

13. Masuda H, Zhang D, Bartholomeusz C, Doihara $H$, Hortobagyi GN and Ueno NT: Role of epidermal growth factor receptor in breast cancer. Breast Cancer Res Treat 136: 331-345, 2012.

14. Gluz O, Liedtke C, Gottschalk N, Pusztai L, Nitz U and Harbeck N: Triple-negative breast cancer - current status and future directions. Ann Oncol 20: 1913-1927, 2009.

15. Corkery B, Crown J, Clynes M and O'Donovan N: Epidermal growth factor receptor as a potential therapeutic target in triplenegative breast cancer. Ann Oncol 20: 862-867, 2009.

16. Wu Q, Ding W, Mirza A, Van Arsdale T, Wei I, Bishop WR, Basso A, McClanahan T, Luo L, Kirschmeier P, et al: Integrative genomics revealed $R A I 3$ is a cell growth-promoting gene and a novel P53 transcriptional target. J Biol Chem 280: 12935-12943, 2005.

17. Tao Q, Fujimoto J, Men T, Ye X, Deng J, Lacroix L, Clifford JL, Mao L, Van Pelt CS, Lee JJ, et al: Identification of the retinoic acid-inducible Gprc5a as a new lung tumor suppressor gene. J Natl Cancer Inst 99: 1668-1682, 2007.

18. Kadara H, Fujimoto J, Men T, Ye X, Lotan D, Lee JS and Lotan R: A Gprc5a tumor suppressor loss of expression signature is conserved, prevalent, and associated with survival in human lung adenocarcinomas. Neoplasia 12: 499-505, 2010.

19. Lin X, Zhong S, Ye X, Liao Y, Yao F, Yang X, Sun B, Zhang J, Li Q, Gao Y, et al: EGFR phosphorylates and inhibits lung tumor suppressor GPRC5A in lung cancer. Mol Cancer 13: 233, 2014.

20. Zhong S, Yin H, Liao Y, Yao F, Li Q, Zhang J, Jiao H, Zhao Y, Xu D, Liu S, et al: Lung tumor suppressor GPRC5A binds EGFR and restrains its effector signaling. Cancer Res 75: 1801-1814, 2015.

21. Sokolenko AP, Bulanova DR, Iyevleva AG, Aleksakhina SN, Preobrazhenskaya EV, Ivantsov AO, Kuligina ES, Mitiushkina NV, Suspitsin EN, Yanus GA, et al: High prevalence of GPRC5A germline mutations in BRCAl-mutant breast cancer patients. Int J Cancer 134: 2352-2358, 2014.

22. Ma T, Yang L and Zhang J: MiRNA-542-3p downregulation promotes trastuzumab resistance in breast cancer cells via AKT activation. Oncol Rep 33: 1215-1220, 2015.

23. Chaffer CL and Weinberg RA: A perspective on cancer cell metastasis. Science 331: 1559-1564, 2011.

24. Lamouille S, Xu J and Derynck R: Molecular mechanisms of epithelial-mesenchymal transition. Nat Rev Mol Cell Biol 15: 178-196, 2014.

25. Cheng Y and Lotan R: Molecular cloning and characterization of a novel retinoic acid-inducible gene that encodes a putative $\mathrm{G}$ protein-coupled receptor. J Biol Chem 273: 35008-35015, 1998.

26. Tao Q, Cheng Y, Clifford J and Lotan R: Characterization of the murine orphan G-protein-coupled receptor gene Rai3 and its regulation by retinoic acid. Genomics 83: 270-280, 2004.

27. Mayer IA, Abramson VG, Lehmann BD and Pietenpol JA: New strategies for triple-negative breast cancer--deciphering the heterogeneity. Clin Cancer Res 20: 782-790, 2014.

28. Taurin S, Allen KM, Scandlyn MJ and Rosengren RJ: Raloxifene reduces triple-negative breast cancer tumor growth and decreases EGFR expression. Int J Oncol 43: 785-792, 2013. 